\title{
VARIABILITY IN THE OUTER PLANET AURORAE
}

\author{
John T. Clarke \\ Department of Atmospheric, Oceanic and Space Sciences, University of Michigan, \\ Ann Arbor, MI 48109-2143, U. S. A.
}

\begin{abstract}
Aurorae on the outer planets have now been detected with ground-based and Earth-orbiting telescopes at far-UV, near-IR, and soft X-ray wavelengths. The best quality observations are of Jupiter, through a combination of very bright aurora and easy spatial discrimination. In this paper the multiwavelength observations will be summarized from the perspective of studying the physical processes and regions of the atmosphere which the different wavelength regions reveal. Sufficient detail is now available in our studies of Jupiter's aurora to begin to draw specific comparisons with processes detected in the Earth's auroral zones, including ionospheric currents, atmospheric heating, and compositional changes.
\end{abstract}

\section{INTRODUCTION}

Observing variability in the outer planet aurorae at present involves observing multi-wavelength emissions, and the relative strengths of these emissions, as well as observing temporal and spatial variations in the emissions of a particular wavelength range. We are now reaching a point when the side effects of the aurora, such as the effect of the auroral energy on the composition and dynamics of the upper atmosphere, can be determined close in time to measurements of the auroral emissions. We hope to arrive soon at a point when changes in the aurora can be observed at the same time as measurements of conditions in the magnetosphere, measured for example by the Galileo orbiter at Jupiter or even from ground-based observations of the Io plasma torus. This paper will begin with an overview comparing the auroral processes on the outer planets, and then discuss in more detail our present understanding of Jupiter's aurora based on multi-wavelength observations.

\section{COMPARING THE OUTER PLANETS}

Jupiter. Jupiter's aurora is extremely bright and energetic compared with any other planet: the average input power in the aurora is believed to be on the order of 1014 Watts. This is sufficient to be by far the dominant source of energy input into Jupiter's thermosphere, exceeding the globally averaged solar EUV input by a factor of 20-50 and dominating the heating and dynamics of Jupiter's thermosphere. Jupiter's auroral zones occur at magnetic latitudes corresponding to the L-shells of the Io plasma torus, although the extent (or lack of) emission toward higher latitudes has not been well established $/ 1$. The auroral zones are characterized by the offset and warped magnetic field geometry of Jupiter, with bright regions at longitudes that correspond to the expected precipitation regions of magnetospheric plasma covering a range of pitch angles and subject to an azimuthal grad-B drift $/ 2$. The direct auroral charged particle excitation of the atmosphere has been observed in the UV and soft $\mathrm{X}$-ray, and secondary atmospheric emissions due to heating, ionization, and compositional changes 
have been observed in the near-IR and thermal $\mathbb{R}$. All of these emissions are observed to vary with time, and to a lesser extent with spatial location.

The composition of the primary particles appears to be limited to energetic electrons plus a lesser but presently unknown contribution from energetic sulfur and oxygen ions $/ 3 /$. There appears to also be considerable excitation from ionospheric currents, including superthermal ionospheric protons $/ 4 /$. The resulting heating of the ionosphere is observed in thermal emission from near-IR lines of $\mathrm{H}_{3}{ }^{+}$ near 2 and $4 \mu \mathrm{m} / 5 /$, and heating of the neutral atmosphere is observed in "hot spots" of $\mathrm{CH}_{4}$ emission near $8 \mu \mathrm{m} / 6 \%$. Somewhat more complicated compositional and thermal changes are observed in lines of $\mathrm{C}_{2} \mathrm{H}_{2}$ and $\mathrm{C}_{2} \mathrm{H}_{6}$ in the thermal $\mathrm{IR} / 7,8 \%$. There is a growing interest in performing simultaneous observations of the UV, IR, and soft X-ray emissions to attempt to sort out the causal relations among the various processes (coordinated through the Jupiter Watch, Auroral Discipline). Jupiter's aurora is described in more detail in the second half of this article.
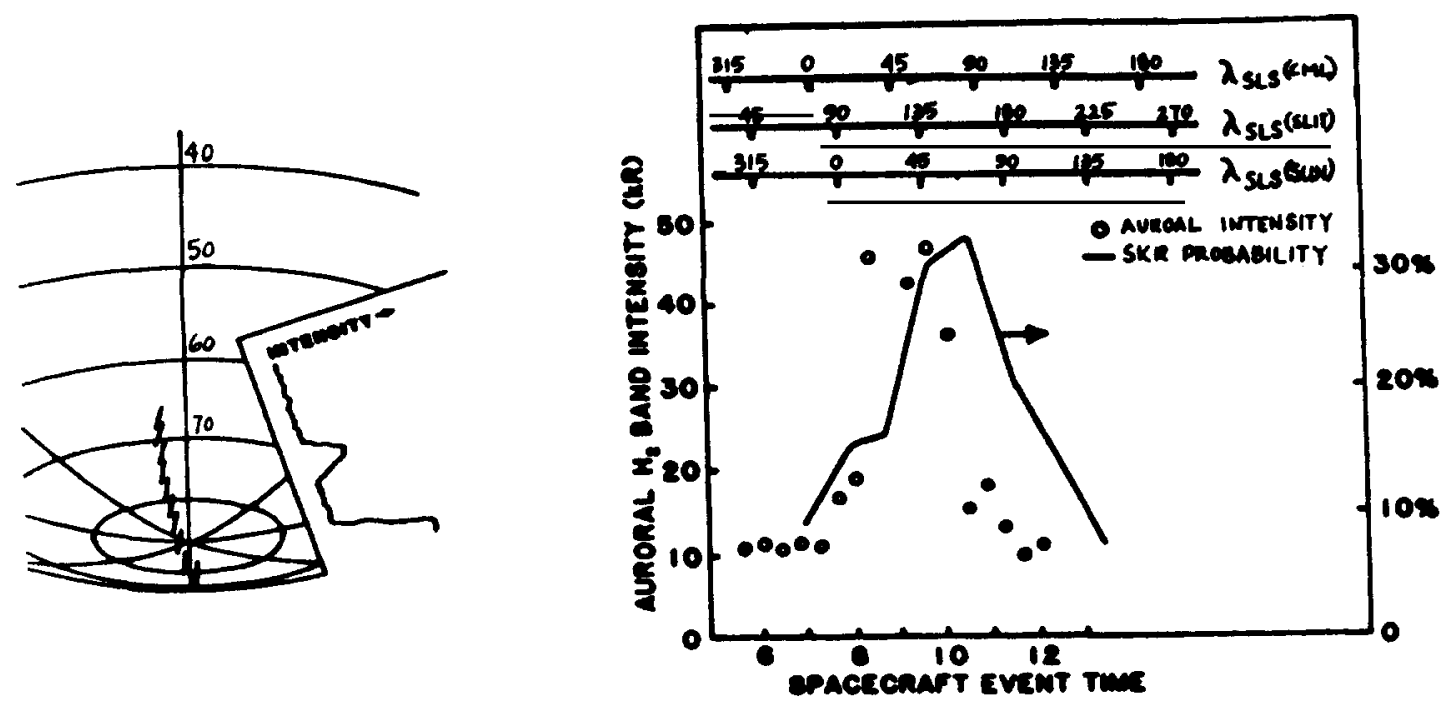

Fig. 1. Plots of Voyager UVS auroral brightness as a function of latitude on Saturn (left), and UV emission intensity and SKR emission probability as a function of Saturnian longitude and sub-solar longitude (right) 1111 .

Saturn. Saturn's aurora is characterized by high latitude $\left(\sim 75-80^{\circ}\right)$ auroral ovals centered on Saturn's aligned magnetic poles (Fig. 1). This aurora has been observed to date only in the UV, although ground-based IR observations show great promise with present capabilities. These IR observations are very important for comparison with the bright Jovian aurora: Jupiter has an atmosphere of similar composition, but a very different magnetospheric environment than Saturn's. Saturn's UV auroral spectrum shows less atmospheric absorption than Jupiter's, suggesting a relatively higher altitude auroral region corresponding (presumably) to less energetic primary particles $19 \%$. The total input power is estimated to be on the order of $10^{11}$ Watts, compared with $10^{14}$ on Jupiter and roughly $10^{10}$ on the Earth. There is some evidence for a higher degree of temporal variability in Saturn's UV auroral brightness than on Jupiter /10/, more like the Earth's aurora than the almost constantly bright Jovian aurora. The high latitude of Saturn's auroral zones indicates that the 
primary particles are precipitating along field lines close to the magnetotail, suggesting an Earth-like auroral process. There is also an apparent correlation between the brightness of the UV aurora and the probability of Saturn kilometric radiation (SKR), which in turn is related to the sub-solar longitude of Saturn (Fig. 1,/11/). This relationship may also indicate an Earth-like auroral process if it means that the orientation of Saturn's magnetic field to the solar wind determines the strength of the aurora.

Uranus. Much less observational data are available for the aurorae on Uranus and Neptune, although UV auroral emissions have been identified on both planets. There is a 4 year time series of IUE observations of the disk-integrated $\mathrm{H}$ Ly $\alpha$ emission from Uranus showing considerable variability, but without any spatial resolution across the planet it is difficult to separate the relative contributions from auroral and airglow processes in these data $/ 12 /$. The relative speed of the variations (up to a factor of 2 increase in less than 8 hours) suggests either an auroral process or an airglow process unlike any of the other planets. Uranus' aurora was also observed by the Voyager 2 UVS near the time of closest approach $/ 13 /$, although the Voyager close encounter appears to have occurred during a relaxation of the magnetosphere following the passage of a solar wind burst when particularly bright aurora would not be expected. Higher spatial resolution maps obtained during the Voyager encounter reveal circumpolar UV auroral ovals closer to the geographic equator than the pole due to the offset and tilted magnetic field /14/. In addition, the weak-field (dayside) auroral oval has a considerably larger radius (roughly $35-40^{\circ}$ ) than the strong-field (nightside) oval (roughly $7-10^{\circ}$ ), consistent with a mapping of L-shells equal to or slightly larger than the Miranda orbit onto poles with roughly an order of magnitude difference in field strength. There is considerable deviation of the observed auroral ovals from calculated constant L-shells, probably due to undetermined higher order moments in Uranus' magnetic field. The total input power in Uranus' aurora is in the broad range of $4 \times 10^{10}$ to $10^{12}$ Watts (comparable to the auroral power on Saturn), with the lower value corresponding to the faint aurora observed by the UVS and the higher value corresponding to the strongest variation observed by IUE. Understanding the driving forces behind Uranus' aurora is particularly interesting at the present epoch, when the combined tilted magnetic field and rotation axis orientation appear to decouple the effects of magnetospheric convection and corotation on the magnetospheric plasma /15/. In this orientation an Earth-like auroral process may proceed unimpeded by the effects of corotation.

Neptune. Neptune as a whole appears fundamentally darker than Uranus in the UV, including any possible auroral emissions. Even the Voyager UVS had difficulty in detecting the low signal levels

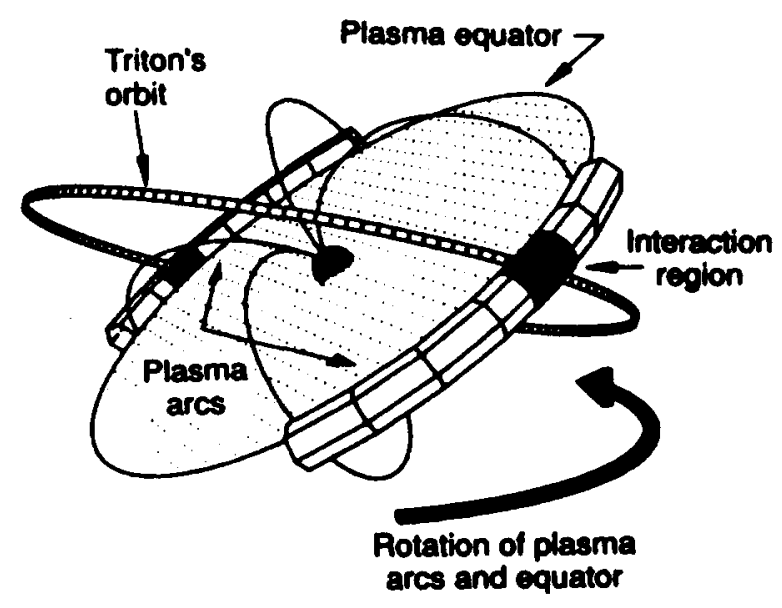

Fig. 2. Schematic model for Neptune auroral precipitation from partial Triton plasma torus /16/. 

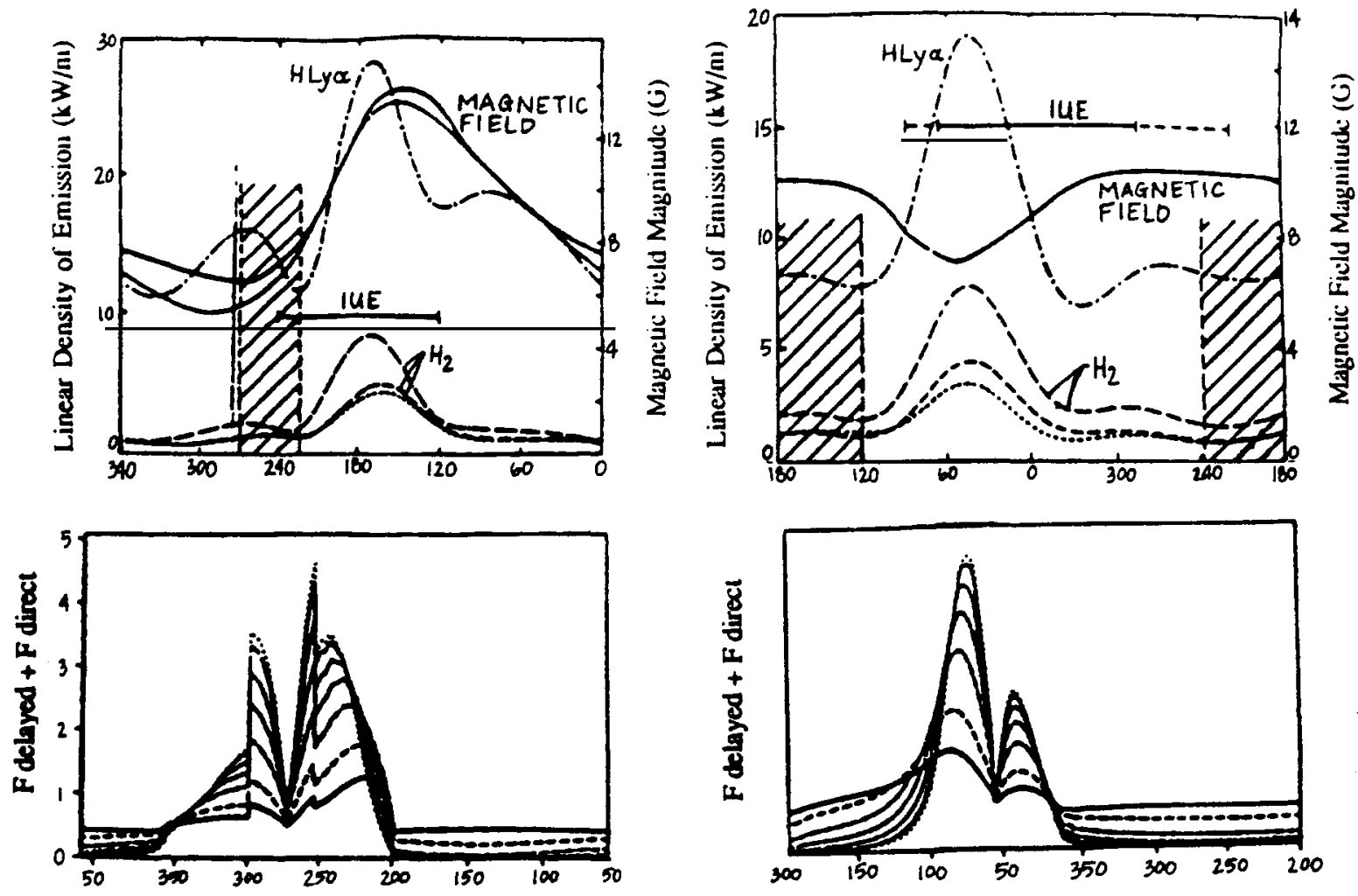

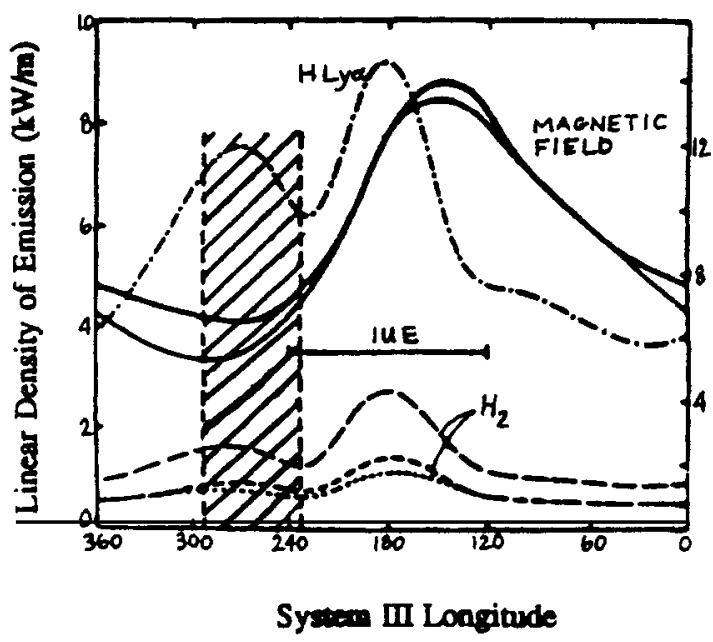

Northern hemisphere

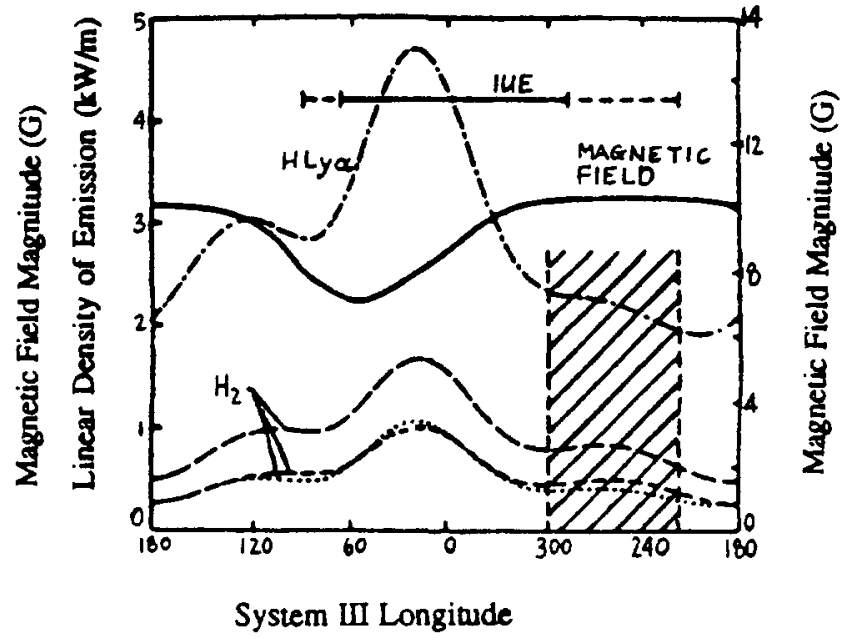

Southern hemisphere

Fig. 3. Observed (IUE and Voyager UVS) and modeled (smooth curves) variations in Jovian auroral UV emission as a function of longiude in the auroral zones $/ 2 \%$. 
from Neptune's aurora near closest approach, although such a detection was made /16/. Faint $\mathrm{H}_{2}$ bands have been identified from the nightside with enhancements near $30^{\circ}$ and $200^{\circ}$ longitude, corresponding to an input power on the order of $10^{9}$ Watts (2-3 orders of magnitude less than Uranus!). These emissions are too faint for variability studies, or even for a good spectral analysis. The longitudinal enhancements have been interpreted in terms of precipitation from a partial Triton plasma torus: the observed auroral longitudes connect magnetically with the two points where Triton's orbit intersects the magnetic equator, where low energy plasma from Triton may be concentrated (Fig. 2, /16/). Lacking any planned deep-space missions to Uranus and Neptune, we must concentrate on the application of remote observations of their aurorae to further our understanding of the magnetospheres of Uranus and Neptune.

\section{JUPITER'S MULTI-WAVELENGTH AURORA}

Observations of Jupiter's aurora in the UV, IR and X-ray have been reviewed /17/: the above section has presented an overview of the earlier results, and the following will concentrate on more recent work.

Distribution of the UV Auroral Ovals. The only direct mapping of the UV auroral ovals has been from Voyager UVS observations of the night atmosphere as Voyager 2 departed the system, during which the aperture was scanned north/south across Jupiter until auroral emission was detected near each pole $/ 1 \%$ This method determined the equatorward extent of the auroral oval as a function of longitude, but gave less information about the extent of the emission toward the pole. The best fit to these data was for an auroral oval at $L=6$, but with some significant deviation from the $\mathrm{O} 4$ magnetic field model. The observed deviations appear within the uncertainty in higher order moments of the magnetic field, which could distort the field geometry near the surface /18/.

Another technique to study the distribution of auroral intensity with longitude, plus any time variations in the emission pattern, has been to observe the variations in UV brightness near the pole with a fixed viewing geometry as the planet rotates. Modeling these observations requires assuming an auroral zone geometry (the $\mathrm{L}=6$ zone described above is consistent with these data) and then varying the emission intensity along the oval. Such modeling has been applied to a decade of IUE observations by $/ 19 /$ and $20 /$, who consistently find UV emission maxima near longitudes of $180^{\circ}$ in the north and $0-500$ in the south. They also find a spectral variation with longitude that suggests a deeper level of auroral emission with respect to hydrocarbon absorption near the emission maxima. This kind of modeling has also been applied to Voyager UVS data with somewhat higher spatial resolution $/ 21$; this modeling reveals a similar but more detailed longitudinal variation. These longitudinal maxima have been theoretically reproduced by $/ 2 /$, who have modeled the drift and precipitation losses of plasma in Jupiter's asymmetric magnetic field (Fig. 3). With considerable independence of the pitch angle distribution assumed, they find that plasma injected near $\mathrm{L}=6$ and subjected to grad-B drift will precipitate into the atmosphere preferentially near the observed longitudes due solely to the asymmetric structure of Jupiter's field. They find offsets in longitude between the modeled and observed UV emission peaks, possibly also indicating higher order moments in Jupiter's magnetic field.

Spectroscopy of UV Auroral Emissions. It is possible to model the depth of the UV auroral emission with respect to the absorbing hydrocarbons (principally $\mathrm{CH}_{4}, \mathrm{C}_{2} \mathrm{H}_{2}$, and $\mathrm{C}_{2} \mathrm{H}_{6}$ ) through comparison of laboratory spectra of electron-excited hydrogen with the observed ratios of the $\mathrm{HLy} \alpha$ and $\mathrm{H}_{2}$ Lyman and Werner band emissions. Modifications of the $\mathrm{H}_{2}$ band ratios indicate mainly the amount of hydrocarbon absorption, since the intrinsic $\mathrm{H}_{2}$ spectrum is nearly independent of electron 
energy above roughly $100 \mathrm{eV}$. The ratio of $\mathrm{H} \mathrm{Ly} \alpha$ to $\mathrm{H}_{2}$ bands may indicate either absorption or the intrinsic ratio of $\mathrm{H} / \mathrm{H}_{2}$ densities, in the sense that the ratio of $\mathrm{H} / \mathrm{H}_{2}$ must increase with altitude from diffusive separation in Jupiter's atmosphere. Existing spectra at 10-30 \& resolution are not well enough resolved to measure the temperature from the vibration/rotation structure of the $\mathrm{H}_{2}$ bands. One serious limitation to this modeling is our knowledge of the atmospheric structure with altitude, which has been measured accurately only near the equator from Voyager UVS occultation data. The expected modifications to the auroral atmosphere by the deposited energy have yet to be taken into account in this modeling.

The spectra of bright and faint northern aurorae have been modeled by $/ 22 /$, who found a similar spectral fit to both the brightest and faintest aurorae on record at that time (Fig. 4). The best fit was obtained for precipitating $95 \mathrm{KeV}$ electrons, which would stop just below the hydrocarbon homopause introducing some absorption of the $\mathrm{H}_{2}$ band emissions. They ruled out the presence of a large population of slightly more energetic particles, and the independence of spectral character on auroral brightness seems to indicate a changing flux of incident particles rather than a changing energy of the beam. The absence of emission lines from oxygen and sulfur in the auroral spectrum has previously been shown to place upper limits on the numbers of precipitating heavy ions in Jupiter's aurora $/ 3 /$.
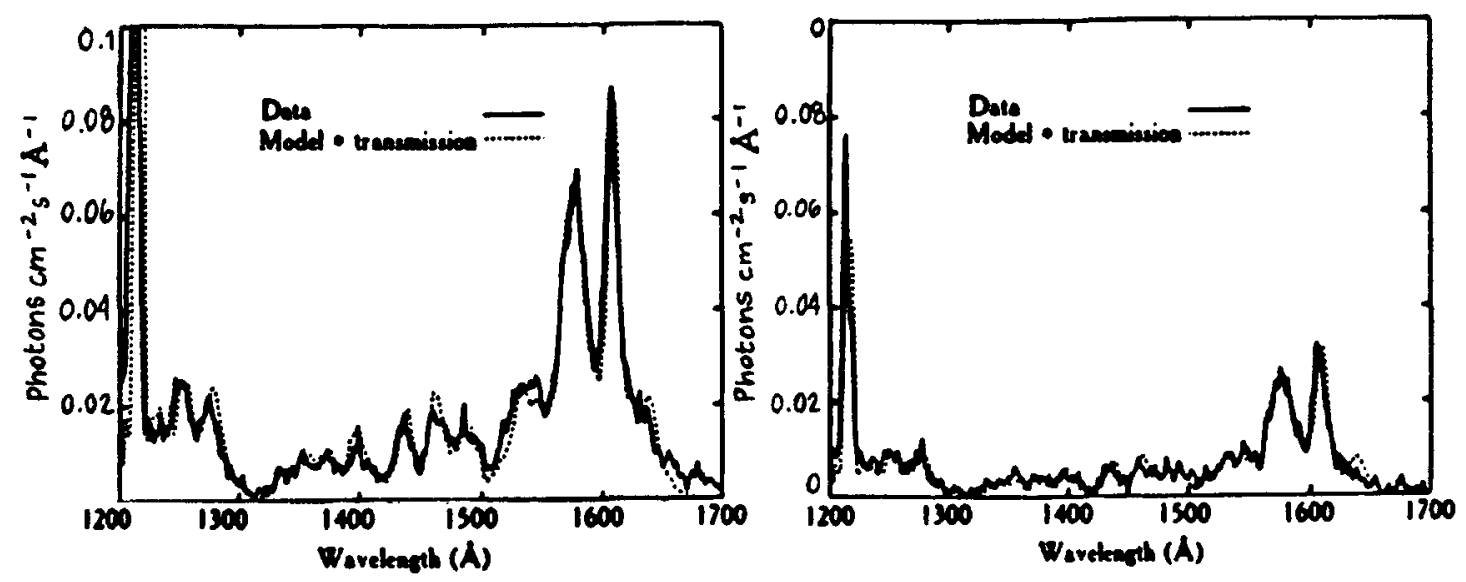

Fig. 4. Observed (smooth curves) and modeled (dashed curves) $\mathrm{UV} \mathrm{H}_{2}$ emission spectra of bright (left) and faint (right) aurora on Jupiter $122 \%$.

Finally, the expected EUV $(\lambda<912 \AA)$ emission spectrum of the aurora has been modeled by $/ 23 /$ in anticipation of the launch of the EUV Explorer spacecraft, which will be capable of high sensitivity spectrometer observations of selected targets. The most probable emissions appear to be those which would be produced by recombination of precipitating oxygen and sulfur ions (Fig. 5). The brightness of these emission lines appears to depend strongly on both the total flux of incoming ions and the depth of penetration (i.e. the incident energy), in the sense that in situ absorption of the emission by $\mathrm{H}_{2}$ will render emissions from deep in the atmosphere difficult to observe. However, observations in the EUV appear to be the best possible way to clearly discriminate between electron and heavy ion precipitation.

Auroral Dynamics and Ion Upwelling. The composition of the precipitating charged particles and the auroral energy budget have been explored through observations of proton excitation of Jupiter's auroral emission. The technique is the same as used for ground-based observations of terrestrial 
aurora, namely to observe Doppler-shifted hydrogen line emission produced through fast proton charge exchange and subsequent radiation. IUE observations at high spectral resolution of Jupiter's aurora have been reported by $/ 4 /$. Whereas during a proton aurora on the Earth a very pronounced

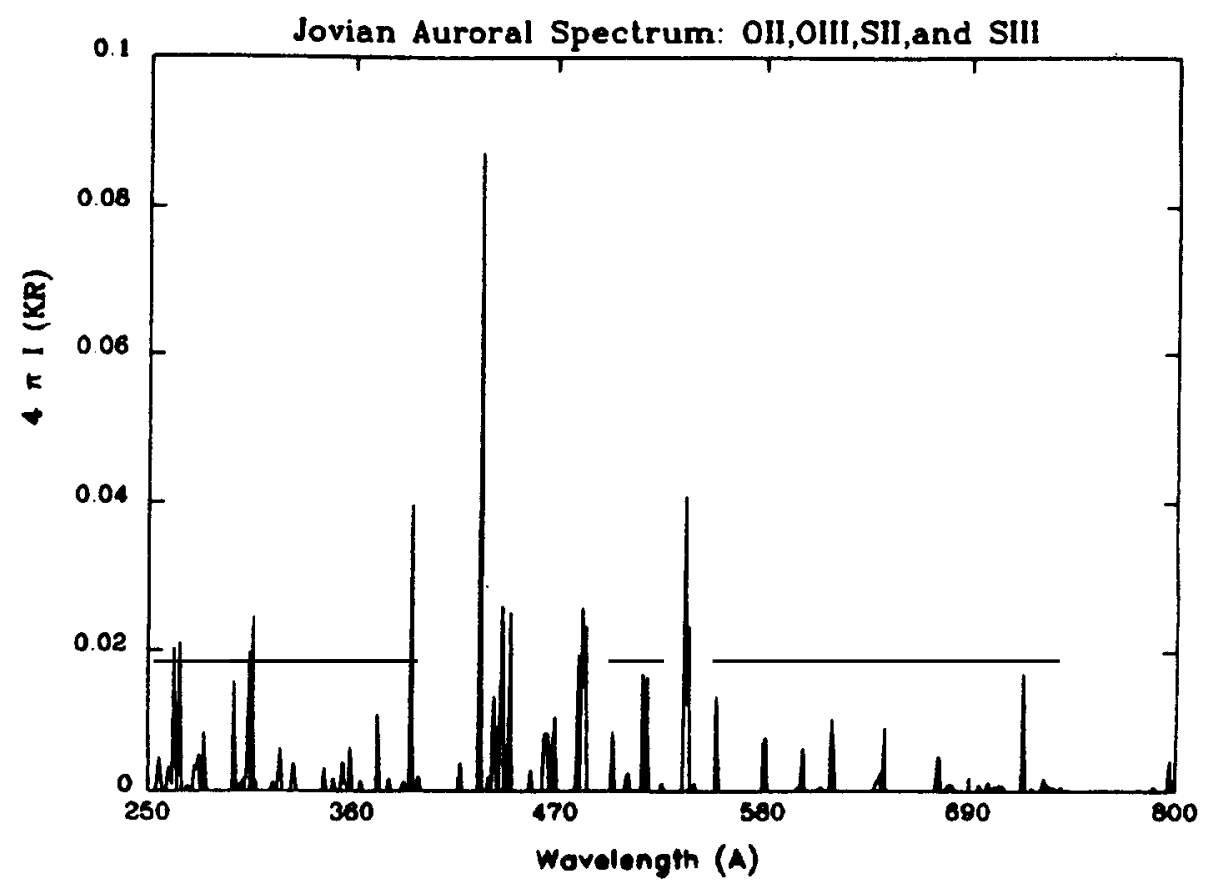

Fig. 5. Modeled EUV emission spectrum of Jupiter's aurora, assuming precipitating $O$ and $S$ ions producing recombination line emission $123 \%$.

Doppler shift and broadening is observed, on Jupiter no comparable effects (for proton energies $E \geq$ $150-200 \mathrm{eV}$ ) have been observed to date (Fig. 6). However, roughly $1 / 2$ of the observed H Ly $\alpha$

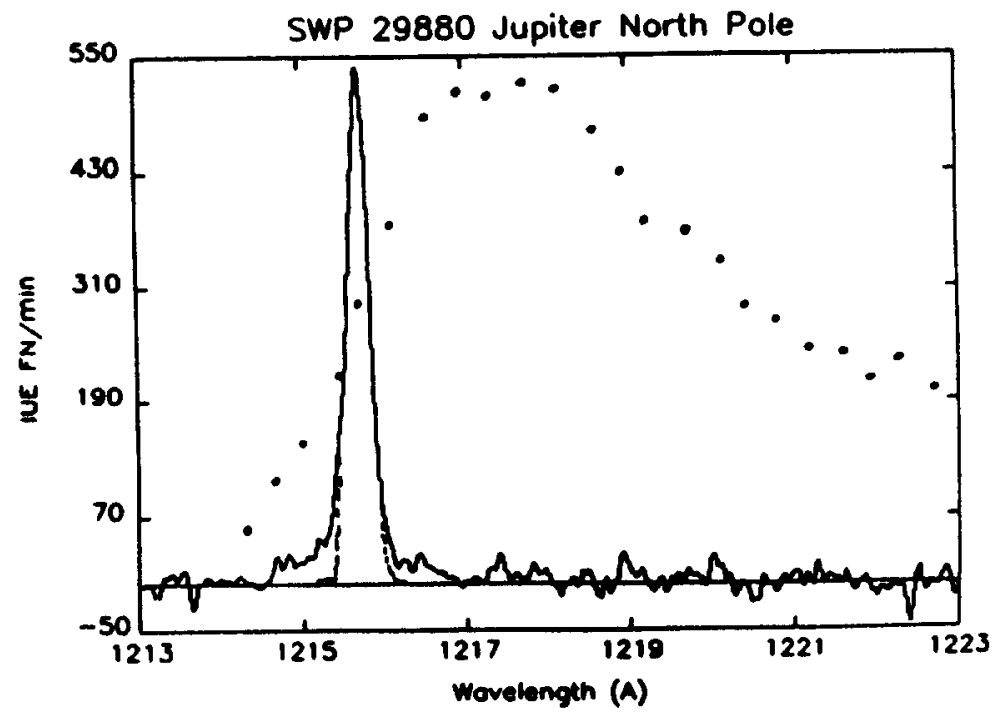

Fig. 6. Observed spectrum of terrestrial $H \alpha$ auroral emission line from proton aurora scaled and reversed to match expected emission from $H$ Ly $\alpha$ from space-based observations (dotted line), and observed $H$ Ly $\alpha$ emission line profile from Jupiter's aurora (solid line) showing a small Doppler broadening above the IUE instrument resolution (dashed line) $14 \%$ 
emission appears Doppler-shifted by a much smaller amount, and predominantly toward the blue on both poles (Fig. 7). More recent spectra of auroral latitudes taken in the absence of bright aurora show un-broadened lines, supporting the identification of this process as auroral in nature. The observed blue shift, representing motion up out of the atmosphere, has been interpreted in terms of acceleration of ionospheric protons, most likely by field-aligned potentials on the order of $10-20 \mathrm{eV}$ total energy. Since charge exchange at these low energies goes predominantly into the ground electronic state, it has been proposed that further collisions of the fast $\mathrm{H}$ atoms with atmospheric neutrals produce the observed emission.
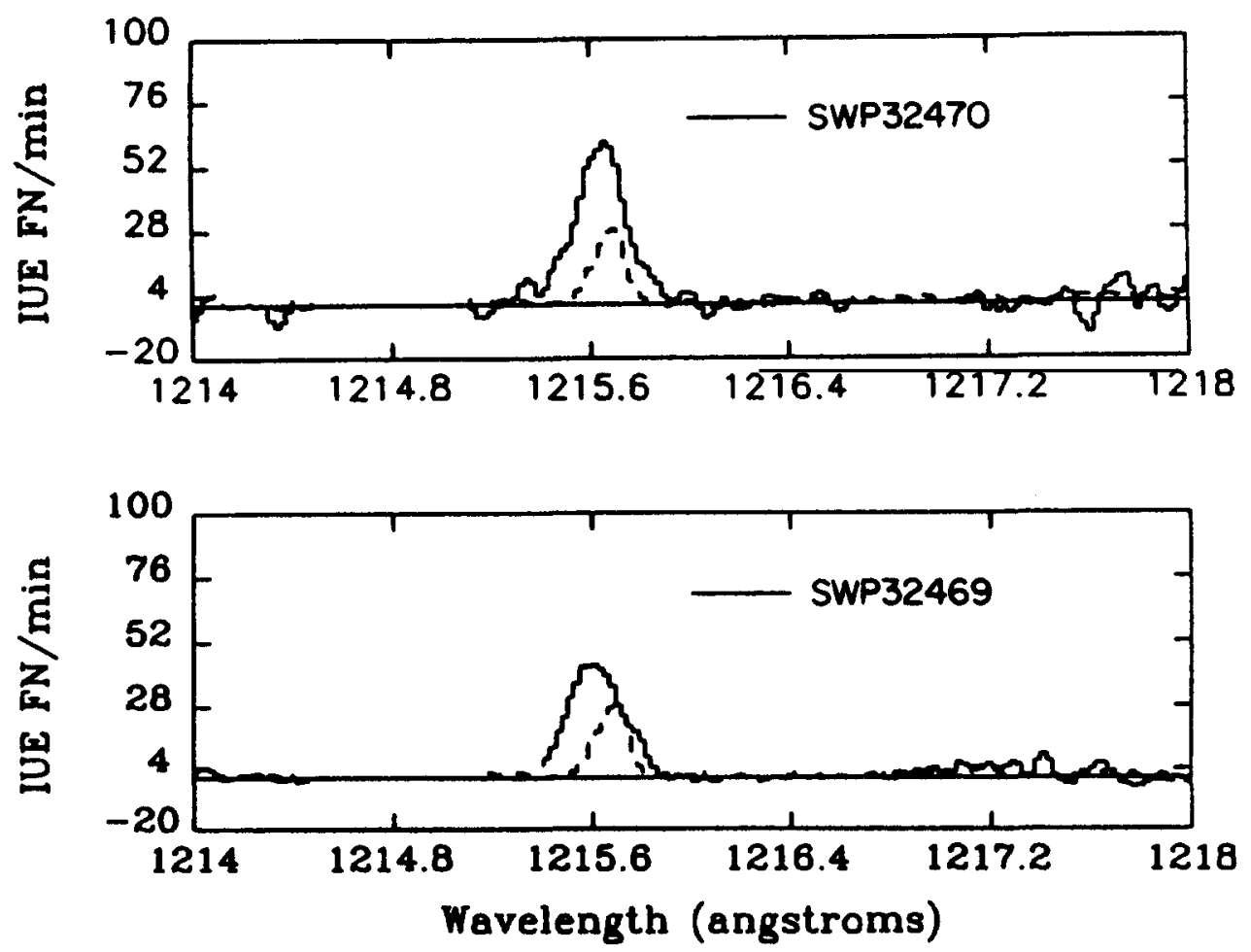

Fig. 7. High resolution IUE spectra of Jovian auroral H Ly $\alpha$ emission in northern (upper plot) and southern (lower plot) aurora (solid lines) compared with width and position of IUE instrument resolution (dashed lines), showing predominantly blue Doppler shifted emission 14/.

The observed Doppler-shifted emissions are on the order of $10 \mathrm{kR}$ in brightness, representing a very large flux of upward moving protons and/or fast $\mathrm{H}$ atoms. The corresponding $\mathrm{H}$ atom velocity is 20 $30 \mathrm{~km} / \mathrm{sec}$, which is a large fraction of the escape velocity (roughly $60 \mathrm{~km} / \mathrm{sec}$ ) and may therefore represent a large flux of ions and/or neutrals into the magnetosphere if the process occurs at sufficiently high altitudes for the particles to escape without further collisions. The hypothesis of a substantial upward flux of plasma from the auroral zones is supported by the identification of 10-100 $\mathrm{eV}$ secondary auroral electrons around $\mathrm{L}=7.5-10$ by the Voyager PLS /24/. A drawing of the expected currents linking the high latitude ionosphere to the Io torus (due to magnetic field ion pickup) is shown in Fig. 8. There may exist additional currents driven by the $\mathbf{J} \times \mathbf{B}$ force resulting from radial diffusion of magnetotail plasma. Following this analogy, it would be expected that there would exist field-aligned currents running both into and out of the Jovian auroral zone at different latitudes: better spatial (latitudinal) resolution will be required to test this idea. In this event there should also exist a strong auroral electrojet that might be observed either directly through Doppler-shifted motions along an appropriate line of sight, or through the east-west motion of the atmosphere resulting from ion drag in the electrojet. Such observations would probably require an orbiting probe, such as Galileo, for the required resolution and line of sight. 


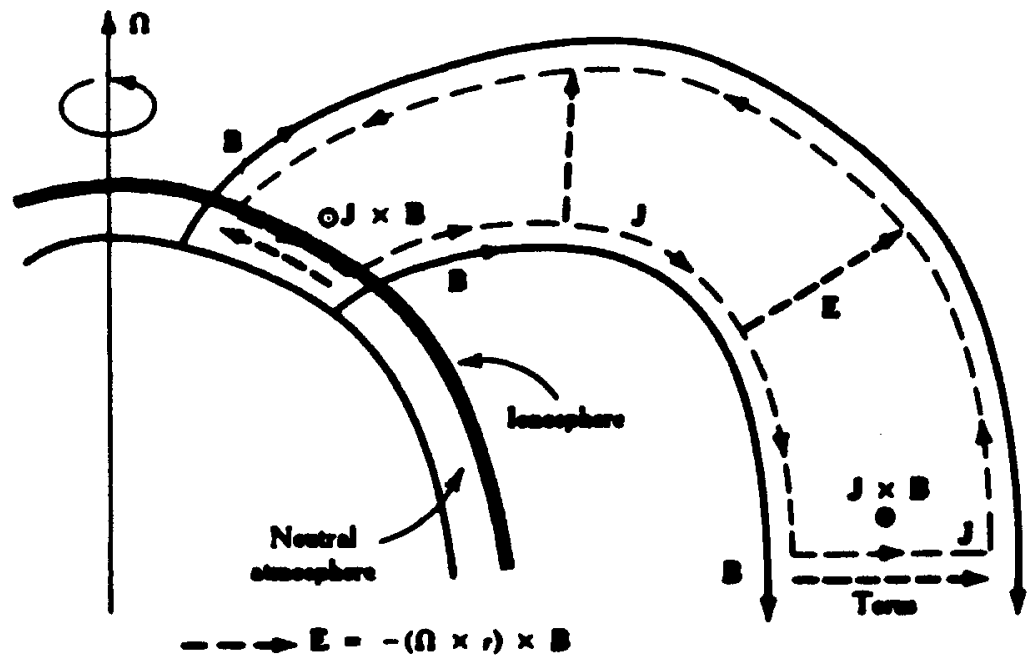

Fig. 8. Drawing of current systems connecting Jupiter's auroral ionosphere to the Io plasma torus 128\%. An additional current system may link higher latitude regions to the magnetotail (see text).

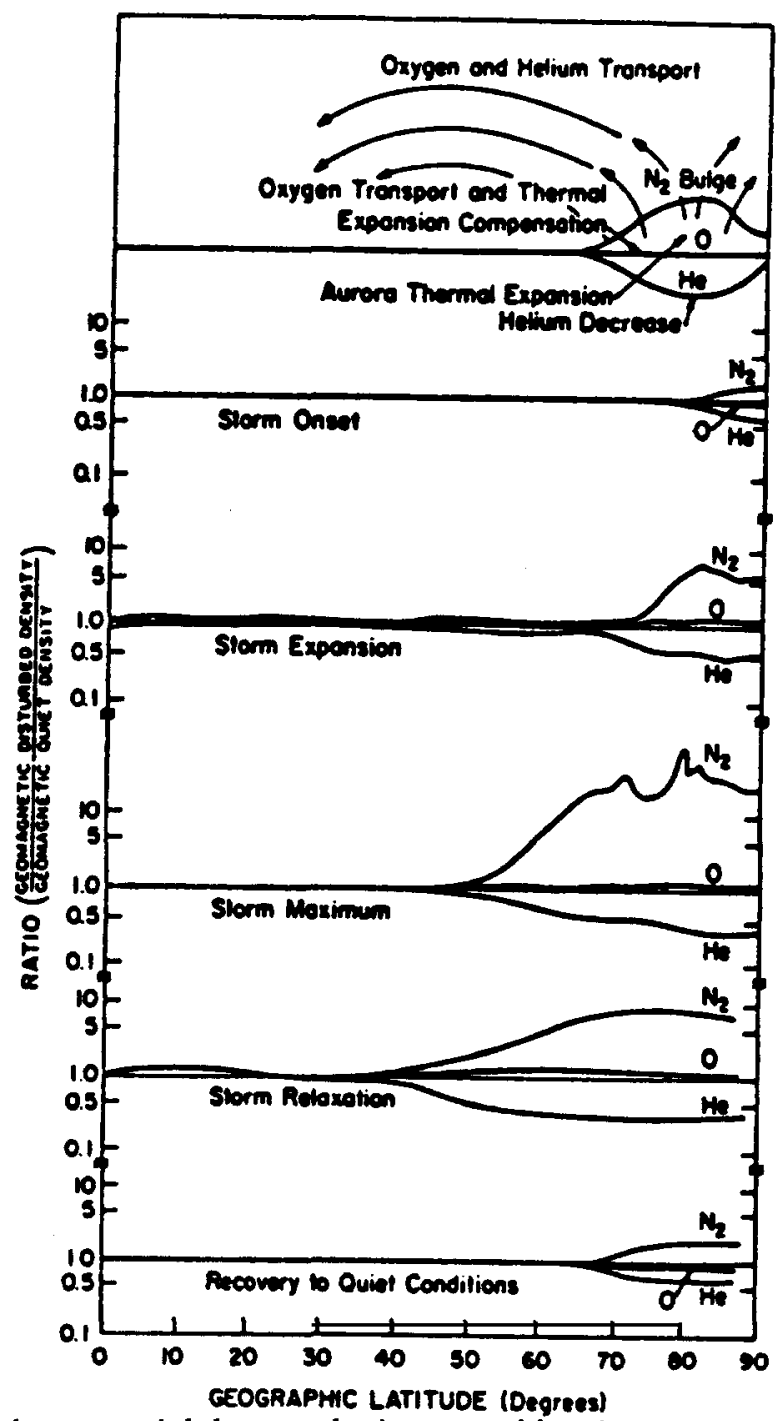

Fig. 9. Schematic of the terrestrial thermospheric compositional response to a geomagnetic storm as a function of latitude at different times during the storm. The ratio is storm-time departure relative to geomagnetically quiet conditions $/ 29 /$. 
IR Emission from Auroral Latitudes. A large number of different IR emissions have now been observed from Jupiter's auroral latitudes, and these observations have the clear advantage of beingpossible from ground-based observatories. The main emissions have been described in the preceding section, along with references to the observations. Of these IR emissions, only the $\mathrm{H} 2$ quadrupole lines near $2 \mu \mathrm{m}$ are believed to be produced by direct charged particle impact $25,26 /$. The other IR emissions fall into one of two categories: emissions whose strength is dominated by the temperature of the emitting species, and emissions whose strength is dominated by the density of the species along the emitting column in the atmosphere. The $\mathrm{CH}_{4}$ emissions at $7.8 \mu \mathrm{m}$ are believed to represent thermal "hot spots" in the upper stratosphere, whereas the emissions of $\mathrm{C}_{2} \mathrm{H}_{2}$ $(13 \mu \mathrm{m}), \mathrm{C}_{2} \mathrm{H}_{6}(12 \mu \mathrm{m})$, and $\mathrm{NH}_{3}(10.3 \mu \mathrm{m}) / 27 /$ are believed to represent mainly density fluctuations in the upper stratosphere. It is of considerable theoretical interest to attempt to model how the auroral energy, which is believed to be deposited well above the altitude of the stratospheric emissions, leads to pronounced thermal and compositional changes at lower altitudes. Once again by analogy to the terrestrial aurora (Fig. 9), the kinds of thermospheric thermal and dynamical perturbations observed in the Earth's aurora should also lead to upwelling and/or heating in the stratosphere on Jupiter.

The observed emissions from $\mathrm{H}_{3}{ }^{+}$near 2 and $4 \mu \mathrm{m} / 5 /$ appear to fall into a different category: being ionic emissions they must be produced in the vicinity of the F-peak, and being rotation-vibration bands they must be sensitive to the ion temperature. Like the $\mathrm{H}_{2}$ quadrupole lines observed at similar wavelengths, these emissions may be produced close in altitude to the directly excited UV aurora and thereby offer a means of studying the direct auroral activity with ground-based telescopes. It is now important to make further simultaneous measurements of the $2-4 \mu \mathrm{m}$ emissions and UV emissions to attempt to establish these correlations.

\section{CONCLUSIONS}

This brief paper has reviewed our knowledge of auroral processes on the outer giant planets, based predominantly on space-based observations over the last decade. Far more information is available about Jupiter's aurora than any other outer planet, and the observational data on Jupiter are sufficient for relatively detailed studies of variability and modeling of some detailed magnetospheric and thermospheric processes. Satum's aurora is possible to study with a fairly straightforward extension of the techniques presently employed for Jupiter, but further studies of the auroral processes on Uranus and especially Neptune will require better capabilities than have previously been employed. On a positive note, the upcoming Galileo orbital tour of Jupiter in combination with rapidly developing remote observing techniques should lead to a greatly improved understanding of Jupiter's highly energetic and variable auroral processes over the next few years.

\section{ACKNOWLEDGEMENT}

This work has been supported by NASA grant NAGW-1766 to the University of Michigan.

\section{REFERENCES}

/1/ A. L. Broadfoot, B. R. Sandel, D. E. Shemansky, J. C. McConnell, G. R. Smith, J. B. Holberg, S. K. Atreya, T. M. Donahue, D. F. Strobel, and J. L. Bertaux, Overview of the Voyager ultraviolet spectrometry results through Jupiter encounter, J. Geophys. Res., 86, 8259 (1981).

/2/ R. Prange and M. Elkhamsi, Modeling the precipitation flux in the Jovian auroral zones, $J$. Geophys. Res., in press (1990).

B/ J. H. Waite, J. T. Clarke, T. E. Cravens, and C. M. Hammond, The Jovian aurora: electron or ion precipitation?, J. Geophys. Res., 93, 7244 (1988). 
/4/ J. T. Clarke, J. Trauger, and H. Waite, Doppler-shifted H Ly $\alpha$ emission from Jupiter's aurora, Geophys. Res. Lett., 16, 587 (1989).

15/ P. Drossart, J. P. Maillard, J. Caldwell, S. J. Kim, J. K. G. Watson, W. A. Majewski, J. Tennyson, S. Miller, S. K. Atreya, J. T. Clarke, J. H. Waite Jr., and R. Wagener , Detection of $\mathrm{H}_{3}{ }^{+}$ on Jupiter, Nature, 340, 6234 (1989).

/6/ J. A. Caldwell, A. T. Tokunaga, and F. C. Gillette, Possible infrared aurora on Jupiter, Icarus, 44,667 (1980).

7/ T. Kostiuk, F. Espenak, M. M. Mumma, D. Deming, and D. Zipoy, Variability of ethane on Jupiter, Icarus, 72, 394, (1987).

/8/ P. Drossart, B. Bezard, S. K. Atreya, J. Lacy, E. Serabyn, A. Tokunaga, and T. Encrenaz, Enhanced acetylene emission near the north pole of Jupiter, Icarus, 66, 610 (1986).

19/ D. E. Shemansky and J. A. Ajello, The Saturn Spectrum in the EUV - Electron excited Hydrogen, J. Geophys. Res., 88, 459, (1983).

/10/ J. T. Clarke, H. W. Moos, S. K. Atreya, and A. L. Lane, IUE detection of bursts of H Ly $\alpha$ emission from Saturn, Nature, 290, 226 (1981).

/11/ B. R. Sandel and A. L. Broadfoot, Morphology of Saturn's aurora, Nature, 292, 679 (1981).

/12/ J. T. Clarke , S. Durrance, S. Atreya, A. Barnes, J. Belcher, M. Festou, C. Imhoff, J. Mihalov, W. Moos, J. Murthy, A. Pradhan, and T. Skinner, Continued observations of the H Ly $\alpha$ emission from Uranus, J. Geophys. Res., 91, 8771 (1986).

/13/ A. L. Broadfoot , F. Herbert, J. B. Holberg, D. M. Hunten, S. Kumar, B. R. Sandel, D. E. Shemansky, G. R. Smith, R. V. Yelle, D. F. Strobel, H. W. Moos, T. M. Donahue, S. K. Atreya, J. L. Bertaux, J. E. Blamont, J. C. McConnell, A. J. Dessler, S. Linick, R. Springer, Ultraviolet spectrometer observations of Uranus,Science, 233, 74 (1986).

/14/ F. Herbert and B. R. Sandel, Characteristics of the Uranian aurora, EOS, Trans. of the Am. Geophys. U, 70, 1174, (1989).

/15/ R. S. Selesnick, Magnetospheric convection in the nondipolar magnetic field of Uranus, $J$. Geophys. Res., 93, 9607 (1988).

/16/ A. L. Broadfoot, S. K. Atreya, J. L Bertaux, J. E. Blamont, A. J. Dessler, T. M. Donahue, W. T. Forrester, D. T. Hall, F. Herbert, J. B. Holberg, D. M. Hunten, V. A. Krasnopolsky, S. Linick, J. I Lunine, J. C. McConnell, H. W. Moos, B. R. Sandel, N. M. Schneider, D. E. Shemansky, G. R. Smith, D. F. Strobel, R. V. Yelle, Ultraviolet spectrometer observations of Neptune and Triton, Science, 246, 1459 (1989).

/17/ J. T. Clarke, J. Caldwell, T. Skinner, and R. Yelle, The aurora and airglow of Jupiter, in Time Variable Phenomena in the Jovian System, NASA SP-494, 211 (1989).

/18/ M. H. Acuna, K. W. Behannon, and J. E. P. Connerney, Jupiter's magnetic field and magnetosphere, Physics of the Jovian Magnetosphere, ed. A.J. Dessler, 1, Cambridge (1983).

/19/ T. E. Skinner, S. T. Durrance, P. D. Feldman, and H. W. Moos, IUE observations of longitudinal and temporal variations in the Jovian auroral emission, Astrophys. J., 278, 441 (1984). 
120/ T. A. Livengood, D. F. Strobel, and H. W. Moos, Long-term Study of longitudinal dependence in primary particle precipitation in the North Jovian aurora, J. Geophys. Res. 95, 10, 375 (1990).

21/ F. Herbert, B. R. Sandel, and A. L. Broadfoot, Observations of the Jovian UV aurora by Voyager, J. Geophys. Res., 92, 3141 (1987).

/22/ G. R. Gladstone and T. E. Skinner, Spectral analysis of Jovian auroral emissions, in Time Variable Phenomena in the Jovian System, NASA SP-494, 221 (1989).

/23/ J. T. Clarke, W. Harris, H. Waite, R. Gladstone, and S. Chakrabarti, Modeled EUV spectrum of Jupiter's aurora, in Extreme Ultraviolet Astronomy, ed. R.F. Malina and S. Bowyer, in press, Pergamon (1990).

124/ R. L. McNutt, F. Bagenal, and R. M. Thome, Observation of Jovian auroral secondary electrons in the Jovian magnetosphere, Geophys. Res. Lett., 17, 291 (1990).

125/ L. Trafton, D. F. Lester, and K. L. Thompson, Unidentified emission lines in Jupiter's northern and southern $2 \mathrm{~mm}$ aurorae, Astrophys. J. Lett., 343, L73 (1989).

126/ S. J. Kim, P. Drossart, J. Caldwell, and J. -P. Maillard, Temperatures of the Jovian Auroral Zone infered from $2 \mathrm{~mm} \mathrm{H}_{2}$ Quadrupole Line Observations, Icarus, 84, 54 (1990).

/27/ T. M. Kostiuk, M. J. Mumma, J. J. Hillman, D. Buhl, L. W. Brown, and J. L. Faris, $\mathrm{NH}_{3}$ spectral line measurements on Earth and Jupiter using a $10 \mu \mathrm{m}$ superheterodyne receiver, Infrared Phys., 1, 431 (1977).

/28/ F. Bagenal, Torus-magnetosphere coupling, in Time-variable phenomena in the Jovian system, NASA SP-494, p. 196 (1989).

129/ A. D. Richmond, Thermospheric dynamics and electrodynamics, Solar-Terrestrial Physics, ed. R.L. Carovillano and J.M. Forbes, 523, D. Reidel (1983). 